\title{
Darstellung des Begriffs Toleranz im Deutschen und im Polnischen aus Frame-semantischer Perspektive
}

\author{
Karina Dudzik (Lublin)
}

\begin{abstract}
The principal aim of this article is to create a cognitive holistic definition of 'tolerance' in Polish and in German based on the approach of the Frame Semantics according to Alexander Ziem (2008). The theory relies on the concept "frame", understood as a cognitive structure facilitating the understanding of linguistic meaning. The semantic frame consists of "slots" and instances of slots, namely "default values" and "fillers". The empirical material includes two types of data: selected dictionary definitions of "tolerance" and some press article containing this notion. It has been carried out a survey among the Polish and the German native speakers to evaluate the particular meaning aspects of "tolerance" i. e. to distinguish between the default values and the fillers of the frame TOLERANCE.
\end{abstract}

\section{$1 \quad$ Einleitung}

Die kognitive Linguistik, ein in den 70er Jahren des 20. Jahrhunderts in den USA entstandener Forschungszweig der Linguistik, befasst sich mit der Beschreibung und Erklärung der mentalen Sprachprozesse und betrachtet die Sprache als Ausdruck der kognitiven Fähigkeit des Menschen. Es wird u. a. untersucht, auf welche Art und Weise menschliches Wissen organisiert ist und welche Struktur es besitzt. Eine der bedeutendsten Theorien ist dabei die FrameSemantik, die von Fillmore (1975) formuliert wurde. Mit dem Begriff „Frame“ beschäftigen sich jedoch nicht nur Linguisten (wie z. B. Konerding 1993; Ziem 2008), sondern auch KIForscher (u. a. Minsky 1975; Charniak 1976), Psychologen (z. B. Barsalou 1992; Schank/Abelson 1997) oder Computerlinguisten (z. B. Peterson 2007).

Das Ziel des vorliegenden Beitrags besteht darin, eine kognitive Definition von Toleranz im Deutschen sowie im Polnischen aus frame-semantischer Sicht zu schildern. Dies folgt unter Verwendung von drei Arten der Korpusdaten, zu denen die Definitionen von Toleranz aus den polnischen und deutschen Wörterbüchern, die polnischen und deutschen Pressetexte sowie eine schriftliche Befragung gehören. Es werden dabei zwei Hypothesen von der Autorin aufgestellt, nämlich: (1) Toleranz kann von den polnischen und deutschen MuttersprachlerInnen unterschiedlich begriffen werden; (2) es können sich für die Benutzer beider Sprachen ganz andere Bedeutungsaspekte von Toleranz als typisch erweisen. Obwohl es eine Vielzahl der von Linguisten bearbeiteten Frame-Konzepte gibt, stützt sich die Autorin nicht umsonst 
auf die Frame-Theorie von Ziem (2008). Seiner Frame-Theorie liegt ein holistisches Modell ${ }^{1}$ der kognitiven Linguistik zugrunde, es wird eine nicht reduktionistische Erfassung der Bedeutung eines sprachlichen Ausdrucks angestrebt, die keinen verstehensrelevanten Bedeutungsaspekt übersehen lässt.

\section{Frame als Analyseinstrument nach Alexander Ziem}

Die Tatsache, dass die SprachbenutzerInnen über Informationen verfügen, die eine lexikographische Definition des jeweiligen sprachlichen Ausdrucks überschreiten, ist leicht zu erklären, denn sprachliche Ausdrücke erwecken bei einem Sprachrezipienten immer ein bestimmtes Hintergrundwissen. Zum einen ist dieses Wissen veränderlich, weil es zahlreichen Modifikationen unterliegt, d. h. es wird ständig aktualisiert und erweitert. Zum anderen ist es verstehensrelevant, man benötigt das Wissen, um einen Ausdruck korrekt zu verstehen und zu gebrauchen. Der Begriff „Wissen“ spielt im Modell von Ziem eine wichtige Rolle. Er geht davon aus, dass „Wissen, das zur Erfassung der Bedeutung eines sprachlichen Ausdrucks eingebracht wird, Strukturen aufweist, die sich mittels Frames linguistisch beschreiben lassen“ (Ziem 2008: 2). Als Frames bezeichnet er „konzeptuelle Wissenseinheiten, die durch sprachliche Ausdrücke beim Sprachverstehen evoziert werden, die also die Sprachbenutzer aus ihrem Gedächtnis abrufen, um die Bedeutung eines sprachlichen Ausdrucks zu erfassen“ (ibd.). Frames dienen zur Strukturierung und Organisation des Wissens, das zum Verstehen eines sprachlichen Ausdrucks relevant ist (cf. Ziem 2008: 281). Frames weisen einen schematischen Charakter auf. Sie enthalten zahlreiche Leerstellen (slots), die sprachlich mittels konkreter Füllwerte (fillers) oder Standardwerte (default values) besetzt werden (cf. Ziem, 2008: 12).

Unter dem Begriff „Leerstellen“ werden mögliche Bezugsstellen verstanden, „hinsichtlich derer sich das Bezugsobjekt eines sprachlichen Ausdrucks prädikativ näher bestimmen lässt" (Ziem 2008: 446). Die Leerstellen haben eine wichtige Funktion zu erfüllen, sie deuten nämlich darauf hin, welche möglichen Prädikate man einem sprachlichen Ausdruck zuschreibt. Diese können in Gestalt von Fragen auftreten, die zu einem bestimmten Bezugsobjekt gestellt werden (ibd.). Zieht man das Wort Auto in Erwägung, kann man nach seiner Farbe, Größe, Marke oder seinen Bestandteilen als Leerstellen fragen. Die Ermittlung von Leerstellen eines Frames erfolgt durch die von Konerding (1993: 139-217) entwickelte Methode der Hyperonymtypenreduktion. Mithilfe von lexikographischen Definitionen wird ein Oberbegriff (Hyperonym) zu dem untersuchten sprachlichen Ausdruck festgestellt und in Anlehnung an dieses Hyperonym eine Liste von Fragen erstellt, die den Leerstellen eines Frames entsprechen. Der schematische Verlauf der Reduktion lässt sich am Beispiel des Ausdrucks Finanzinvestor (cf. Ziem, 2008: 415) wie folgt veranschaulichen:

\section{Finanzinvestor $\rightarrow$ Investor $\rightarrow$ jemand $\rightarrow$ Person}

Für den Ausdruck Finanzinvestor bildet Investor einen Oberbegriff. Investor ist laut Definition im Duden Deutsches Universalwörterbuch (1989) ,jmd., der anlegt (...)“. Das aus

\footnotetext{
${ }^{1}$ Im Rahmen der kognitiven Linguistik entstand neben einem holistischen Ansatz noch ein entgegengesetzter, der modularistische Ansatz, u. a. von Monika Schwarz (1992) und Manfred Bierwisch (1983) repräsentiert.
}

ISSN 1615-3014 
der lexikographischen Definition extrahierte jemand ist als Hyperonym für Inverstor zu verstehen. Der Oberbegriff Person ist wiederum das Endglied der Hyperonymtypenreduktion.

Die Leerstellen werden mit zwei unterschiedlichen Werten, nämlich Standardwerten und konkreten Werten ausgefüllt. In Bezug auf das vorher genannte Beispiel mit Auto kann ein durchschnittlicher Sprachbenutzer, in dem er auf seinem Wissen und seinen Lebenserfahrungen basiert, die Fragen beantworten, aus welchen Teilen ein typisches Auto besteht, wozu es dient oder wer das Auto fahren kann. Dies bedeutet, dass er diesem Bezugsobjekt gewisse Prädikate zuschreibt, selbst wenn sie im Text (abgesehen von seiner geschriebenen oder gesprochenen Form) nicht auftauchten (cf. Ziem 2008: 10-11). Diese Art impliziter Prädikationen werden Standardwerte genannt. Sie werden als Annahmen der SprachbenutzerInnen verstanden, die bei der Aktivierung eines Frames aus dem Gedächtnis abgerufen werden (cf. Ziem 2008: 247). Darüber hinaus verweist Ziem (2008: 33) darauf, dass ,oftmals nicht ein einziger Standardwert eine Leerstelle besetzt, sondern es eine Vielzahl gleichwertiger Möglichkeiten gibt". Es wäre noch zu erläutern, wie Standardwerte eigentlich entstehen. Hinsichtlich des Ausdrucks Auto sind die SprachbenutzerInnen imstande, mehr als eine Farbe oder Größe des Autos anzugeben. Linguistisch heißt das: Sie haben viele Prädikate zur Verfügung, mit denen sie die Leerstellen ,Funktion' oder ,Größe' des Frames AUTO füllen können. Manche benutzen sie häufiger, manche dagegen werden von ihnen kaum verwendet. Wenn ein Prädikat häufiger als ein anderes vorkommt, gewinnt es an Salienz, verfestigt sich und dadurch kann es künftig zu einem Standardwert werden (cf. Ziem 2008: 341). Dieser Prozess verläuft jedoch in zwei Richtungen. Wird ein Prädikat immer seltener verwendet, kann es in Zukunft nicht mehr als Standardwert angesehen werden und wird durch andere Prädikate ersetzt.

Um die dritte Art von Strukturkonstituenten des Frames, nämlich konkrete Füllwerte zu erklären, lohnt es sich, ein interessantes Beispiel aus der F.A.Z (06.08.2008) heranzuführen. Einer der Zeitungsartikel beginnt folgendermaßen: „'Sprechendes Auto“ soll Straßen sicherer machen. Wenige Unfälle und Staus: Dafür soll die ,intelligente Fahrzeugkommunikation“ sorgen“. Stoßen die LeserInnen auf Sätze, in denen über sprechende Autos berichtet wird, wissen sie sofort aufgrund der Lebenserfahrung, dass es sich eher um eine neue Erfindung von Autokonstrukteuren als um eine typische Eigenschaft von Autos handelt. Im Gegensatz zu den Standardwerten, die auf dem Vorwissen beruhen, spricht man von konkreten Füllwerten, wenn „Leerstellen durch Daten der aktuellen Wahrnehmung besetzt werden“ (Ziem 2008: 267). Konkrete Füllwerte legen die Spezifizierungen eines Frames dar, die im Text ausdrücklich gegeben werden (cf. Ziem 2008: 13). Sie treten in Form von expliziten Prädikationen auf. Ihre Verwendung von SprachbenutzerInnen geht immer mit der Perspektivierung des Referenzobjektes einher. Mit expliziten Prädikationen werden bestimmte Wissensaspekte hervorgehoben, während andere in den Hintergrund rücken (cf. Ziem 2008: 330-334).

Eine deutliche und überzeugende Feststellung wird im Rahmen der KI-Forschung vorgeschlagen, wenn es sich um die Unterschiede zwischen Standardwerten und konkreten Füllwerten handelt. Die Standardwerte resultieren aus Erwartungen der SprachbenutzerInnen, während konkrete Füllwerte unmittelbar aus Daten folgen (cf. Strube et al. 1996: 110, 161, zitiert nach Ziem 2008: 267). 


\subsection{Korpusdaten}

Zwei Arten von Textdaten, d. h. Wörterbücher und Presseartikel sind Bestandteile des polnischen und des deutschen Untersuchungskorpus. Sie haben während der Analyse unterschiedliche Rolle zu erfüllen. Die Definitionen von „Toleranz“ aus den Wörterbüchen werden von der Autorin als lexikalische Basis angesehen. Auf ihrer Grundlage entstanden die für beide Sprachen gleichen Vorlagen des Fragebogens mit dem Ziel, den Stellenwert einzelner Bedeutungsvarianten von Toleranz für die deutschen und polnischen MuttersprachlerInnen zu untersuchen. Die Autorin geht jedoch von der Annahme aus, dass diese Definitionen zwar detailliert und vielseitig, aber nicht erschöpfend sind. Es lassen sich mit Sicherheit solche Bedeutungskomponenten von Toleranz auffinden, die im Wörterbuch nicht berücksichtigt werden, aber von großem Rang sind. In die Analyse werden deswegen auch Presseartikel einbezogen, weil sie neue Bedeutungsaspekte enthalten, die Vielseitigkeit des untersuchten Begriffs dokumentieren und zur Erstellung einer nicht reduktionistischen Definition von Toleranz beitragen.

Bei der Korpuskonstitution wird das Kriterium der Ausgewogenheit als grundlegend angenommen. Weder qualitativ noch quantitativ sollen die Teilkorpora $\mathrm{zu}$ sehr voneinander abweichen, wenn man im weiteren Teil des Beitrags Vergleiche zwischen den kognitiven Definitionen von Toleranz in beiden Sprachen anstellen will.

Das deutsche Untersuchungskorpus machen 4 Wörterbücher und 30 Presseartikel aus. Zu den deutschen Wörterbüchern gehören Der Knaur. Universallexikon in 14 Bänden (1992), Deutsches Wörterbuch (2002), Duden Deutsches Universalwörterbuch (2003) sowie Duden Herkunftsbücher der deutschen Sprache (1989). Der Analyse werden auch 30 Pressetexte unterzogen. Die Mehrheit von ihnen (22 Artikel) kommt aus dem Spiegel. Den übrigen Teil bilden 5 Texte aus der Süddeutschen Zeitung (SZ) und 3 Texte aus der Frankfurter Allgemeinen Zeitung (F.A.Z.).

Die kognitive Definition von Toleranz wird im Polnischen unter Verwendung von Uniwersalny słownik języka polskiego (2003), Praktyczny słownik współczesnej polszczyzny (2003), Słownik etymologiczny języka polskiego (2005) und Wielki słownik języka polskiego (2008) aufgestellt. Die zweite Gruppe der Textdaten stellen 30 polnische Presseartikel dar. Die größte Anzahl von Texten (14 Artikel) kommt aus Newsweek. Aus Rzeczpospolita (Rz) werden 10 Texte entnommen. Für die Analyse werden auch 6 Artikel aus Gazeta wyborcza $(G W)$ benutzt.

Die Korpora werden nach folgenden Kriterien zusammengestellt: Im Falle der polnischen und deutschen Wörterbücher konzentriert sich die Autorin auf die relativ neuen Werke. Fast alle berücksichtigten Wörterbücher entstanden nach dem Jahre 2000. Für die Presseartikel ${ }^{2}$ gelten zwei wichtige Kriterien. Alle polnischen und deutschen Pressetexte werden aus den Zeitungen entnommen, die landesweit vertrieben werden und die sich einer hohen Auflage erfreuen. Auch das relativ breite Meinungsspektrum wird dann mit diesem Korpus abgedeckt. Was die

\footnotetext{
2 Alle Pressetexte sind in elektronischer Form verfügbar und werden aus den Internetausgaben der Zeitungen heruntergeladen.
} 
temporale Einschränkung der Korpusquellen anbetrifft, stammen die Texte aus dem Zeitraum 2013 bis 2015, weil erstens die aktuelle Verwendung des Begriffs „Toleranz“ untersucht werden sollte und zweitens das Jahr 2015 als Abschluss der Korpuszusammenstellung galt. Die Korpusquellen lieferten zu dieser Zeit nur zirka 30 Texte (in jeder Sprache) mit den entsprechenden Korpusbelegen.

\subsection{Methodologisches Verfahren}

Es muss am Anfang hervorgehoben werden, dass das Analysemodell ${ }^{3}$ von Ziem (2008: 406421) für die Autorin des vorliegenden Beitrags ein fundamentaler Ausgangspunkt für die Erstellung ihres eigenen Modells ist. Die Modelle decken sich zwar in manchen Punkten miteinander, von der Autorin werden aber neue Etappen des methodologischen Verfahrens hinzugefügt. Die Analyse verläuft mithilfe von zwei Arten der Textdaten, während bei Ziem nur Pressetexte das Untersuchungskorpus ausmachen. Außerdem wird eine Umfrage durchgeführt (sie kommt bei Ziem nicht vor), um die Standardwerte des Frames TOLERANZ zu ermitteln. Es ist deshalb sinnvoll, das vollständige methodologische Verfahren schrittweise zu präsentieren:

\section{Annotation der Textbelege:}

Im Falle der deutsch- und polnischsprachigen Wörterbücher werden die Definitionen von Toleranz in ihrer Ganzheit der Analyse unterzogen. Im Gegensatz dazu wird bei jedem der Pressetexte der Umfang des zu untersuchenden Kontextes separat bestimmt. Es handelt sich um die Textausschnitte, in denen die Ausdrücke Toleranz/tolerancja, Intoleranz/nietolerancja, tolerieren/tolerować sowie ihre anderen Wortformen oder Komposita (wie z. B. Toleranzgrenze/granica tolerancji) vorkommen.

\section{Bestimmung der Leerstellen:}

Selbst wenn die früher erwähnte Hyperonymtypenreduktion als Methode zur Gewinnung eines Oberbegriffs und zur Feststellung der Leerstellen eines Frames von vielen Linguisten (u. a. Konerding, 1993; Ziem, 2008; Czachur, 2011) erfolgreich angewandt wird, kann sie sich bei manchen sprachlichen Ausdrücken (einschließlich der von der Autorin analysierten Toleranz) als problematisch erweisen. Im Duden Online-Wörterbuch werden drei Bedeutungsvarianten von Toleranz ${ }^{4}$ angegeben:

1. das Tolerantsein, Duldsamkeit;

2. begrenzte Widerstandsfähigkeit des Organismus gegenüber schädlichen äußeren Einwirkungen, z. B. gegenüber Giftstoffen oder Strahlen;

\footnotetext{
${ }^{3}$ Die Analyse von Alexander Ziem hatte zum Ziel, Standardwerte der Metapher Heuschrecke zu bestimmen. Fünf wichtige Etappen der Analyse sind aufzuzählen: (1) Annotation der Textbelege: Sammlung der entsprechenden Anzahl von Texten, in denen die Metapher Heuschrecke vorkommt. Für die Texte werden erstens eine bestimmte Zeitspanne, zweitens der Umfang des zu untersuchenden Kontextes bestimmt. (2) Prädikationsanalyse: Gewinnung von Prädikationen, die die Metapher anbetreffen, und ihre Darstellung in Form von vereinfachten Sätzen. (3) Hyperonymtypenreduktion: Gewinnung des Hyperonyms (Organismus) zu dem untersuchten Ausdruck Heuschrecke mithilfe von Duden Universalwörterbuch (1989). Auflistung der in Klassen thematisch eingeteilten Prädikatoren. (4) Klassifikation expliziter Prädikationen: Zuordnung expliziter Prädikationen zu einem bestimmten Prädikator. (5) Auswertung der Ergebnisse.

${ }^{4} \mathrm{http}: / / \mathrm{www} . d u d e n . d e /$ rechtschreibung/Toleranz [25.01.2015].
} 
3. zulässige Differenz zwischen der angestrebten Norm und den tatsächlichen Maßen, Größen, Mengen usw.

Für jede einzelne Bedeutungsvariante wird von der Autorin der Versuch unternommen, einen Oberbegriff (Hyperonym) festzustellen. Schematisch lässt sich dieses Verfahren folgendermaßen präsentieren:

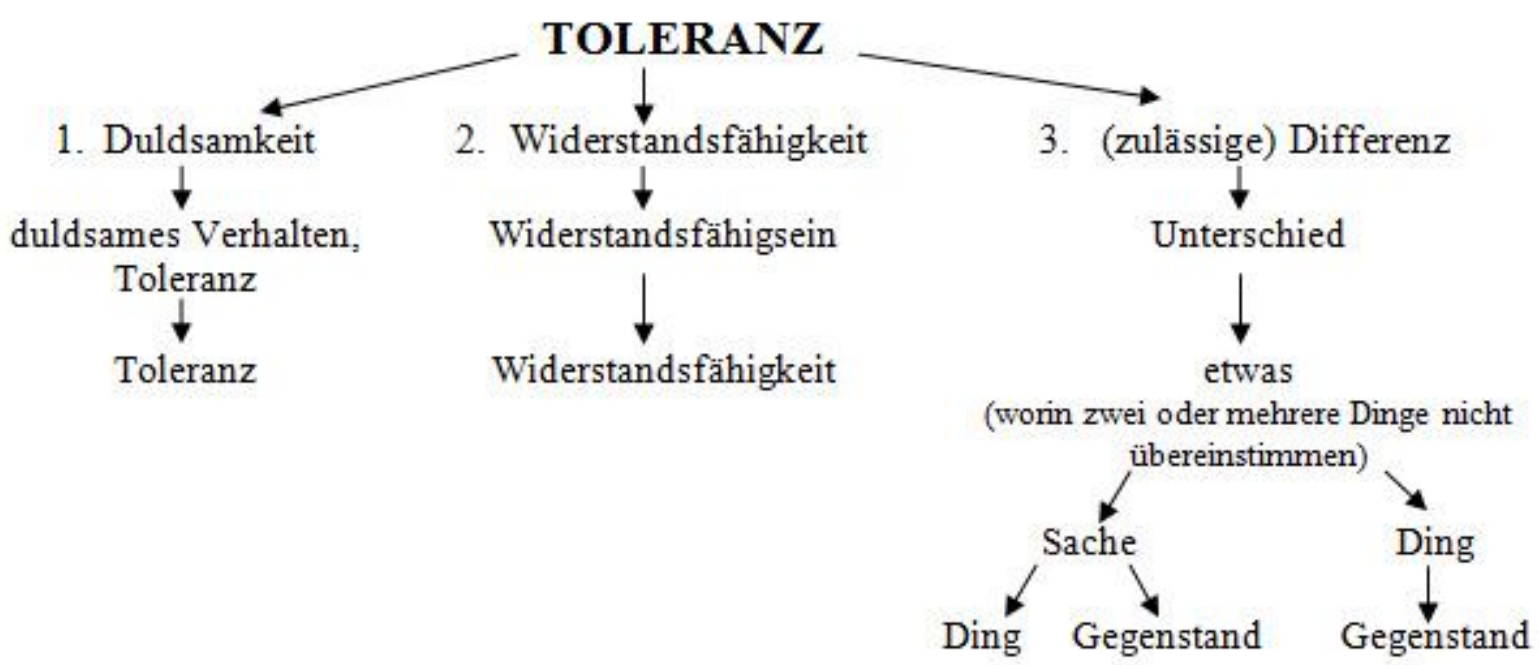

Abbildung 1: Schematische Hyperonymtypenreduktion am Beispiel des Begriffs ,Toleranz“

Da der Test, alle Bedeutungsvarianten von Toleranz auf ein gemeinsames Hyperonym zurückzuführen, fehlschlägt, wird von der Autorin eine Liste mit Fragen erstellt, die aus den Valenzstellen resultieren und den Leerstellen des Frames TOLERANZ entsprechen. Die Vorgehensweise wird an einem Beispiel der Definition ${ }^{5}$ von Toleranz aus dem Deutschen Wörterbuch (2002) präsentiert, nämlich:

1) Duldung, zunächst im religiösen Bereich (...);

2) Rücksichtnahme auf Andersdenkende, Akzeptieren anderer Meinungen;

3) (...) zulässige Abweichung von einem bestimmten Sollwert; in der Medizin (...) Widerstandsfähigkeit des menschlichen Organismus (...).

Mithilfe von dieser Definition lassen sich folgende Fragen stellen:

- Welche Synonyme gibt es für das Wort Toleranz? (z. B. Duldung, Akzeptieren usw.)

- Was kann man tolerieren? (z. B. andere Meinungen)

- Wen kann man tolerieren? (z. B. Andersdenkende)

Nach der Analyse aller Definitionen werden insgesamt sieben Fragen formuliert: (1) Wer ist zur Toleranz (nicht) fähig? (2) Was ist zur Toleranz (nicht) fähig? (3) Wen kann man (nicht) tolerieren? (4) Was kann man (nicht) tolerieren? (5) Welche anderen Bezeichnungen beziehen sich auf Toleranz? (6) Wo kann (keine) Toleranz stattfinden? (7) Durch welche Eigenschaften kennzeichnet sich Toleranz?

\section{Umfrage:}

\footnotetext{
${ }^{5}$ Form mit Auslassung der Beispiele.
} 
Als ein zusätzlicher Schritt wird die Umfrage in das methodologische Verfahren einbezogen, zum einen um die Konzeptualisierung des Begriffs „Toleranz“ in beiden Sprachen zu untersuchen, zum anderen um die am Anfang gestellte Hypothese hinsichtlich des ungleichen Stellenwertes einzelner Bedeutungsvarianten von Toleranz zu verifizieren. Das Ziel der schriftlichen Befragung besteht in der Bestimmung der Standardwerte, d. h. der im Gedächtnis der SprachbenutzerInnen verfestigen Wissensaspekte des Frames TOLERANZ. An der Umfrage - sie wurde Anfang 2015 durchgeführt - beteiligten sich je 50 polnische und deutsche MuttersprachlerInnen, überwiegend die Studierenden der Katholischen Universität in Lublin und der Universität Regensburg. Der Fragebogen umfasst zwei Fragen: (1) „Was verstehen Sie unter dem Begriff ,Toleranz“?“(2) „Wie kann man das Wort Toleranz ersetzen?“ Der Fragebogen enthält im Falle der ersten Frage eine Auflistung von unterschiedlichen Bedeutungsvarianten von Toleranz und in Bezug auf die zweite Frage synonymische Ausdrücke zu Toleranz, die aus den Wörterbüchern entnommen werden. Die Vorlagen für die Fragebögen in beiden Sprachen sind inhaltlich homogen. Bei jeder Bedeutungsvariante in der ersten Frage sowie bei jedem Synonym zu Toleranz in der zweiten Frage markieren die Befragten nach Ermessen eine Antwort: ,,ja/nein/schwer zu sagen“ und können ebenfalls unter jeder Frage eigene Bemerkungen und andere Bedeutungsvarianten angeben. Bei der Auswertung der Umfrageergebnisse wird ein relevantes Kriterium angewandt: eine Information kann man für einen Standardwert des Frames erklären, wenn mindestens 3/4 der Befragten die Antwort ja markieren. 


\section{Fragebogen}

Das Ziel des vorliegenden Fragebogens besteht in der Gewinnung von Informationen, wie der Begriff Toleranz von den polnischen und deutschen Sprachbenutzern verstanden wird. Bei jeder Feststellung markieren Sie bitte eine der drei möglichen Antworten (ja/nein/schwer zu sagen). Die punktierten Stellen dienen zur Angabe freier Antworten. Der Fragebogen wurde in der polnischen und deutschen Sprache angefertigt und ist anonym

\section{Was verstehen Sie unter dem Begriff Toleranz?}

- Respekt vor einer anderen politischen Gesinnung

- Respekt vor einer anderen sexuellen Orientierung

- Respekt vor einer anderen Religion

- Rechtlich zugelassene Differenz im Feingehalt einer geprägten Münze

- Fähigkeit des Organismus, eine große Dosis der Medikamente oder des Giftes ohne Schaden zu ertragen

- Anerkennung jedes Menschen für einen möglichen Partner des Dialogs (im religiösen Sinne)

- Fähigkeit der Fotoemulsion zum Ausgleichen der Belichtungsfehler

- Nachsicht in Bezug auf ein unangebrachtes Verhalten einer Person

- Zulässige Abweichung von einem geforderten Wert (z. B. Länge oder Gewicht)

- eine von Juden entrichtete Steuer, die sie vom Militärdienst befreite

- Resistenz des Organismus z. B. gegen Temperatur

- Akzeptanz der Antigene im Körper

- Ertragen von unterschiedlichen Missständen, z. B. Drogensucht

\section{Wie kann man den Begriff Toleranz ersetzen?}

- verständnisvoll sein

- akzeptieren

- Nachsicht haben

- ertragen

- zustimmen

- achten

\section{JA NEIN Schwer zu}

sagen

\begin{tabular}{|l|l|l|}
\hline & & \\
\hline & & \\
\hline & & \\
\hline & & \\
\hline & & \\
\hline & & \\
\hline & & \\
\hline & & \\
\hline & & \\
\hline & & \\
\hline & & \\
\hline
\end{tabular}

\begin{tabular}{|l|l|l|}
\hline & & \\
\hline & & \\
\hline & & \\
\hline & & \\
\hline & & \\
\hline
\end{tabular}

Abbildung 2: Die Vorlage des deutschen Fragebogens 


\section{Analyse und Klassifikation expliziter Prädikationen:}

Aus den zur Analyse herangezogenen polnischen und deutschen Pressetexten werden in diesem Schritt alle Prädikationen, und zwar konkrete Füllwerte gewonnen, die den Frame TOLERANZ charakterisieren. Die Autorin listet sie in Form von einfachen Sätzen oder nonverbalen Phrasen auf und ordnet sie den entsprechenden Leerstellen zu. Die Prädikationen aus der polnischen Presse werden ins Deutsche übersetzt.

Diese Herangehensweise illustriert das folgende Beispiel. In einem der Artikel liest man: „In Brüssel sorgt das Vorgehen der türkischen Regierung gegen Journalisten für Unmut. In einer Resolution beklagte das europäische Parlament eine "abnehmende Toleranz" der türkischen Regierung gegenüber öffentlichem Protest und kritischen Medien.“ (Spiegel, 15.01.2015). Aus diesem kurzen Abschnitt resultieren explizit vier Prädikationen:

1. Türkische Regierung ist zur Toleranz fähig;

2. Öffentlicher Protest wird toleriert;

3. Kritische Medien werden toleriert;

4. Toleranz kann abnehmen.

Die erste Prädikation wird dann der Leerstelle „Wer/Was ist zur Toleranz nicht fähig?“ zugeordnet, zwei weitere beziehen sich auf die Leerstelle „Wen/Was kann man (nicht) tolerieren?“ und die letzte Prädikation gehört der Leerstelle „Durch welche Eigenschaften kennzeichnet sich Toleranz?" an.

\section{Auswertung der Ergebnisse:}

Die Endphase besteht darin, eine kognitive Definition von Toleranz in beiden Sprachen zu ermitteln. Die Definitionen aus den Wörterbüchern werden erweitert, indem die aus den Presseartikeln gewonnenen konkreten Füllwerte berücksichtigt werden. Danach folgt der Vergleich zwischen der polnischen und deutschen Definition von Toleranz.

\section{Aufstellung einer kognitiven Definition von Toleranz im Deutschen und im Polni- schen}

\subsection{Begriff „Toleranz“ in den Wörterbüchern}

Die der Analyse unterzogenen Definitionen von Toleranz im Deutschen und im Polnischen unterscheiden sich voneinander je nach Wörterbuch in der Länge sowie in der Anzahl der präsentierten Bedeutungsvarianten. Während in Dem Knaur. Universallexikon in 14 Bänden (1992) fünf Bedeutungsvarianten dargelegt werden, ist im Duden Herkunftsbücher der deutschen Sprache (1989) nur die knappe Feststellung „Duldsamkeit, großzügige Geisteshaltung“ zu lesen. Nicht alle Wörterbücher beschränken sich außerdem auf die Angabe der Bedeutungsaspekte von Toleranz, manche enthalten auch Beispielssätze, sie präsentieren auch den Gebrauch des Ausdrucks Toleranz im Kontext.

Aus den vier Wörterbüchern wurden insgesamt sieben Bedeutungsvarianten von Toleranz entnommen, wobei die drei ersten Varianten in jedem der genannten Wörterbücher vorkommen: 
1. Respekt vor anderen Ansichten, Gefühlen, Gesinnungen und Verhaltensweisen;

2. Resistenz des Organismus (in bestimmten Grenzen) gegen biologische, chemische und physikalische Faktoren;

3. Zulässige Abweichung z. B. der Länge oder des Gewichtes von einem geforderten Wert (im technischen Sinne);

4. eine von Juden entrichtete Steuer, die sie vom Militärdienst befreite;

5. rechtlich zugelassene Differenz im Feingehalt einer geprägten Münze;

6. Fähigkeit der Fotoemulsion zum Ausgleichen der Belichtungsfehler;

7. Anerkennung jedes Menschen für einen möglichen Partner des Dialogs (im religiösen Sinne).

Im Gegensatz dazu wurden in den deutschen Wörterbüchern nur fünf Bedeutungsvarianten des Begriffs „Toleranz“ angegeben. Eine der Bedeutungsvarianten (Nr. 5) kommt nur einmal in Dem Knaur. Universallexikon in 14 Bänden (1992) vor.

1. Duldsamkeit, Geltenlassen anderer Meinungen und Anschauungen;

2. Zulässige Differenz zwischen der angestrebten Norm und den tatsächlichen Maßen, Größen, Mengen o.Ä./zulässige Abweichung von einem gefordertem Wert, Bereich zwischen einem oberen und unteren Grenzwert, innerhalb dessen einwandfreie Funktion des Objektes noch gewährleistet ist;

3. Begrenzte Widerstandsfähigkeit des Organismus gegenüber schädlichen äußeren Einwirkungen;

4. Zulässige Abweichung einer Münze vom gesetzlich vorgeschriebenen Feingehalt und Gewicht;

5. Wirkungsabfall des Medikamentes als Antwort des Körpers nach wiederholter Gabe.

\subsection{Ergebnisse der Umfrage}

Die Ergebnisse der Umfrage wurden berechnet und in Prozent ausgedrückt. Jede der Fragen wurde separat präsentiert und besprochen. Die Unterschiede zwischen der polnischen und der deutschen Definition lassen sich aufweisen, indem die Ergebnisse der Befragungen ${ }^{6}$ nebeneinander geschildert werden:

Was verstehen Sie unter dem Begriff Toleranz?

- Respekt vor einer anderen politischen Gesinnung

- Respekt vor einer anderen sexuellen Orientierung

- Respekt vor einer anderen Religion

- rechtlich zugelassene Differenz im Feingehalt einer geprägten Münze

- Fähigkeit des Organismus, eine große Dosis der Medikamente oder des Giftes ohne Schaden zu ertragen

\begin{tabular}{|c|c|c|}
\multicolumn{1}{|l}{ JA NEIN } & $\begin{array}{l}\text { Schwer } \\
\text { zu } \\
\text { sagen }\end{array}$ \\
\hline $\mathbf{7 6} \%$ & $10 \%$ & $14 \%$ \\
\hline $\mathbf{9 0} \%$ & $8 \%$ & $2 \%$ \\
\hline $\mathbf{9 0} \%$ & $8 \%$ & $2 \%$ \\
\hline $34 \%$ & $44 \%$ & $22 \%$ \\
\hline $56 \%$ & $34 \%$ & $10 \%$ \\
\hline
\end{tabular}

TAK NIE Trudno powiedzieć

\begin{tabular}{|c|c|c|}
\hline $\mathbf{9 4} \%$ & $4 \%$ & $2 \%$ \\
\hline $\mathbf{9 2} \%$ & $8 \%$ & - \\
\hline $\mathbf{1 0 0} \%$ & - & - \\
\hline $32 \%$ & $32 \%$ & $36 \%$ \\
\hline $60 \%$ & $30 \%$ & $10 \%$ \\
\hline
\end{tabular}

\footnotetext{
${ }^{6}$ Die links präsentierte Tabelle bezieht sich auf die Befragung unter den deutschen MuttersprachlerInnen, rechts befinden sich die Ergebnisse der polnischen Umfrage.
} 


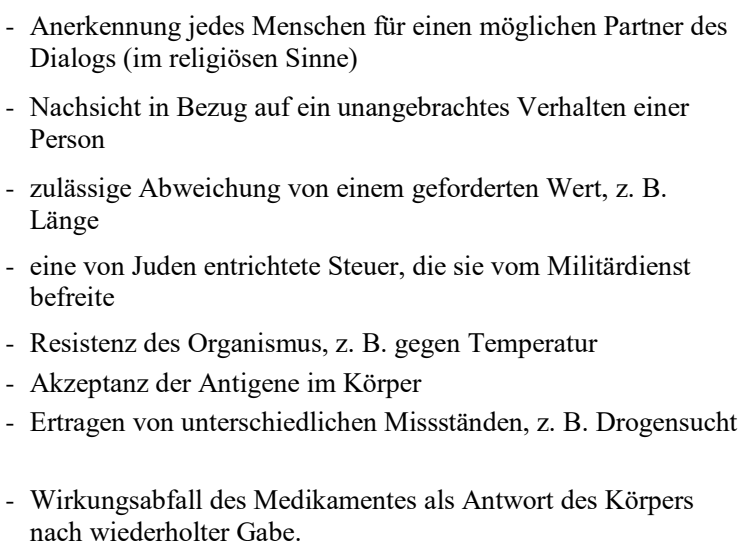

\begin{tabular}{|c|c|c|}
\hline $58 \%$ & $18 \%$ & $24 \%$ \\
\hline $62 \%$ & $18 \%$ & $20 \%$ \\
\hline $\mathbf{9 6} \%$ & - & $4 \%$ \\
\hline $12 \%$ & $62 \%$ & $26 \%$ \\
\hline $34 \%$ & $50 \%$ & $16 \%$ \\
\hline $36 \%$ & $44 \%$ & $20 \%$ \\
\hline $16 \%$ & $48 \%$ & $34 \%$ \\
\hline $20 \%$ & $41 \%$ & $39 \%$ \\
\hline
\end{tabular}

\begin{tabular}{|c|c|c|}
\hline $68 \%$ & $14 \%$ & $18 \%$ \\
\hline $18 \%$ & $74 \%$ & $8 \%$ \\
\hline $48 \%$ & $44 \%$ & $8 \%$ \\
\hline $4 \%$ & $78 \%$ & $18 \%$ \\
\hline $70 \%$ & $22 \%$ & $8 \%$ \\
\hline $52 \%$ & $26 \%$ & $22 \%$ \\
\hline $18 \%$ & $70 \%$ & $12 \%$ \\
\hline $26 \%$ & $40 \%$ & $34 \%$ \\
\hline
\end{tabular}

Tabelle 1: Ergebnisse der polnischen und der deutschen Befragung: Frage Nr. 1.

Mithilfe von dieser Frage wurde geprüft, was die SprachbenutzerInnen unter dem Begriff tolerancja/Toleranz verstehen. Gemäß dem früher festgelegten Kriterium lassen sich in der polnischen Sprache drei Standardwerte des Frames TOLERANZ nachweisen, weil in drei Fällen mehr als $75 \%$ der Befragten die Angaben bejahten. Zu den Standardwerten des Frames TOLERANZ gehören:

1. Toleranz als Respekt vor anderen religiösen Anschauungen (100 \%);

2. Toleranz als Respekt vor einer anderen politischen Gesinnung (94\%);

3. Toleranz als Respekt vor einer anderen sexuellen Orientierung (92\%).

Es ist noch darauf aufmerksam zu machen, dass nicht alle übrigen Bedeutungsvarianten von Toleranz den gleichen Rang für die Befragten besaßen. Während 70 \% der Personen bejahten, dass die Toleranz „die Resistenz des Organismus, z. B. gegen Temperatur“ bedeutet, markierten nur $4 \%$ der Befragten die Antwort $j a$ bei der Angabe „Toleranz als eine von Juden entrichtete Steuer, die sie vom Militärdienst befreite“. Darüber hinaus trugen zwei Personen ihre eigenen Antworten ein. Dazu gehörten „Toleranz des Organismus gegenüber den Nahrungsstoffen“ und „Akzeptanz des Rechtes auf Glück anderer Menschen“.

Im Deutschen wurden dagegen vier Standardwerte des Frames TOLERANZ ermittelt, die sich zum Teil mit den Ergebnissen der polnischen Umfrage decken. Dazu zählt man:

1. Toleranz als zulässige Abweichung von einem geforderten Wert, z. B. Länge oder Gewicht (96\%);

2. Toleranz als Respekt vor einer anderen sexuellen Orientierung (90\%);

3. Toleranz als Respekt vor einer anderen Religion (90\%);

4. Respekt vor einer anderen politischen Gesinnung (76\%).

Was die übrigen konkreten Füllwerte des Frames TOLERANZ im Deutschen anbetrifft, nimmt einer im Vergleich mit anderen einen hohen Platz ein, denn $62 \%$ der befragten Personen bezeichneten den Begriff „Toleranz“ als „Nachsicht bezüglich eines unangemessenen Verhaltens von jemandem“. Im Gegensatz dazu gehört die Bedeutungsvariante „Toleranz als eine von Juden entrichtete Steuer“ zu den weniger typischen konkreten Füllwerten. Diese Antwort gaben nur $12 \%$ der Befragten an. Bei der ersten Frage lassen sich darüber hinaus einige freie Antworten der Befragten aufzählen. Ihrer Ansicht nach bedeutet Toleranz: „libe- 
rale Einstellung gegenüber anderen Menschen und ihrer Lebensweise“, „geistige Offenheit“, „Mangel an Egoismus und reiner Selbstbezogenheit“ sowie „Anerkennung der Überzeugungen anderer Menschen, obwohl sie möglicherweise von eigenen Überzeugungen und Vorstellungen abweichen“".

Dass der Begriff „Toleranz“ von polnischen und deutschen MuttersprachlerInnen unterschiedlich konzeptualisiert wird, ist auch in der zweiten Frage sichtbar. Mithilfe dieser Frage wurde geprüft, mit welchen Verben und Ausdrücken sich das Verb tolerieren ersetzen lässt. Die Befragten gaben die folgenden Antworten an:

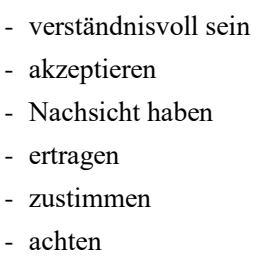

\begin{tabular}{|c|c|c|}
\hline $\mathbf{8 0} \%$ & $12 \%$ & $8 \%$ \\
\hline $68 \%$ & $16 \%$ & $16 \%$ \\
\hline $52 \%$ & $14 \%$ & $34 \%$ \\
\hline $60 \%$ & $28 \%$ & $12 \%$ \\
\hline $6 \%$ & $72 \%$ & $22 \%$ \\
\hline $68 \%$ & $16 \%$ & $16 \%$ \\
\hline
\end{tabular}

\begin{tabular}{|c|c|c|}
\hline $\mathbf{8 2} \%$ & $16 \%$ & $2 \%$ \\
\hline $\mathbf{9 0} \%$ & $8 \%$ & $2 \%$ \\
\hline $14 \%$ & $78 \%$ & $8 \%$ \\
\hline $50 \%$ & $46 \%$ & $4 \%$ \\
\hline $26 \%$ & $52 \%$ & $22 \%$ \\
\hline $\mathbf{9 4} \%$ & $4 \%$ & $2 \%$ \\
\hline
\end{tabular}

Tabelle 2: Ergebnisse der polnischen und der deutschen Befragung: Frage Nr. 2.

Es wurden drei Standardwerte im Polnischen festgestellt:

1. achten $(94 \%)$

2. akzeptieren $(90 \%)$

3. verständnisvoll sein $(82 \%)$

Alle übrigen Synonyme wurden als konkrete Füllwerte des Frames TOLERANZ betrachtet. Es ist auch zu beachten, dass sich das Verb „ertragen“ für die Befragten als problematisch erwies. Die Hälfte der Personen hielt „tolerieren" und „ertragen“ für Synonyme, während 46\% (fast die Hälfte) behaupteten, dass sich die Bedeutungen dieser Verben miteinander nicht decken. Obwohl in allen ausgewählten polnischen Wörterbüchern der Ausdruck ,, Nachsicht haben“ als Synonym für „tolerieren“ vorkommt, wurde dies in dieser Umfrage nicht bestätigt.

Im Deutschen wurde dagegen nur ein synonymischer Ausdruck als Standardwert des Frames TOLERANZ festgestellt, der Ausdruck ,verständnisvoll sein“ wurde von $80 \%$ aller Befragten für Synonym zu ,tolerieren“ erklärt.

Im Vergleich zu übrigen konkreten Füllwerten belegen die Verben ,akzeptieren“ (68\%) und „achten“ (68 \%) eine hohe Position. Für $72 \%$ der Befragten erwiesen sich die Verben ,tolerieren“ und „zustimmen“ als keine Synonyme. Zehn Personen gaben außerdem eigene freie Antworten an. Ihrer Ansicht nach lässt sich das Verb ,tolerieren“ durch solche Synonyme wie „respektieren“ (2 Personen), „dulden“ (2 Personen), „offen sein“ (1 Person), „unvoreingenommen sein“ (1 Person), ,gewähren lassen“ (1 Person), ,gelten lassen“ (1 Person), ,,aufgeschlossen sein“ (1 Person) und ,aushalten“ (1 Person) ersetzen.

\subsection{Analyse der Presseartikel}

Aus den Pressetexten gewonnene konkrete Füllwerte des Frames TOLERANZ werden nachfolgend den entsprechenden Leerstellen zugeordnet. Zum einen aus Platzgründen, zum anderen zwecks besserer Veranschaulichung konkreter Füllwerte des Frames TOLERANZ wurden 
sie in der Weise geschildert, dass sich in jeder der Tabellen konkrete Füllwerte ${ }^{7}$ aus den polnischen Presseartikeln links und konkrete Füllwerte aus den deutschen Pressetexten rechts befinden.

Die erste Tabelle enthält die Antworten auf die Frage, wer oder was zur Toleranz (nicht) fähig ist. Insgesamt wurden in den polnischen Pressetexten 24 und in den deutschen 14 explizit ausgedrückte Füllwerte markiert:

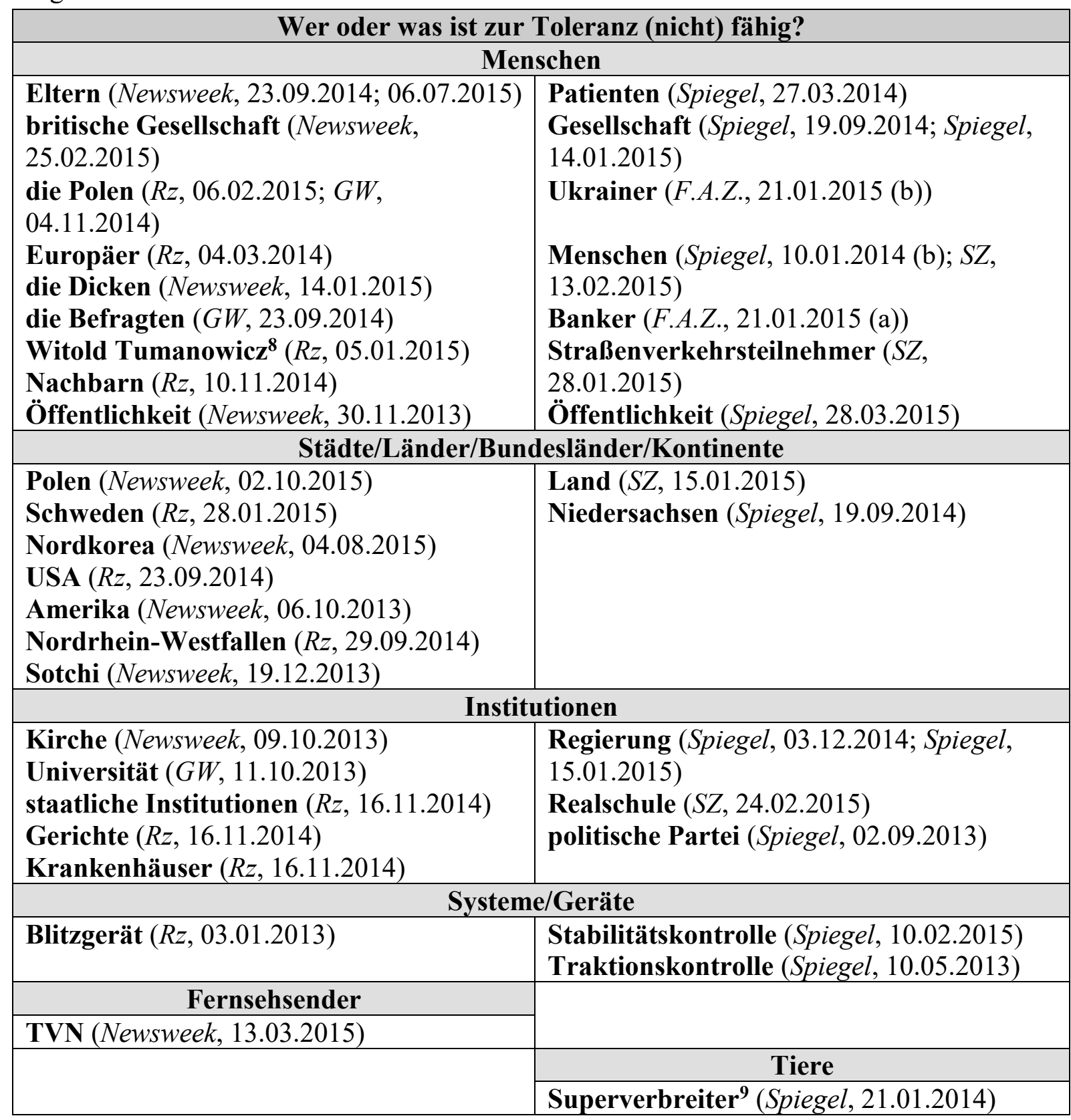

Tabelle 3: Konkrete Füllwerte der Leerstelle „Wer oder was ist zur Toleranz (nicht) fähig?““ auf der Basis von den polnischen und den deutschen Presseartikeln.

\footnotetext{
7 Die präsentierten konkreten Füllwerte sowie die im Kap. 5 zitierten Textabschnitte aus der polnischen Presse wurden von der Autorin ins Deutsche übersetzt.

${ }^{8}$ Leiter des Marsches für Unabhängigkeit.

${ }^{9}$ In diesem Kontext sind Mäuse gemeint.
} 
Konkrete Füllwerte, die sich auf die Leerstelle, wen oder was man (nicht) tolerieren kann, beziehen, wurden in der zweiten Tabelle präsentiert. In Bezug auf diese Frage wurde die größte Anzahl der Antworten, nämlich 30 deutsche und 31 polnische Füllwerte notiert.

Wer oder was kann (nicht) toleriert werden?

\begin{tabular}{|c|c|}
\hline \multicolumn{2}{|c|}{ Menschen } \\
\hline $\begin{array}{l}\text { die Transsexuellen }(G W, 03.06 .2014) \\
\text { die Transgender }(G W, 03.06 .2014) \\
\text { die Transvestiten }(G W, 03.06 .2014) \\
\text { Mitglieder der LGBT }(G W, 03.06 .2014 \text {; } \\
\text { Newsweek, 23.09.2014) } \\
\text { Christen }(\text { Newsweek, } 04.08 .2015) \\
\text { Anhänger des Islams }(\text { Newsweek, } \\
25.02 .2015) \\
\text { anders Liebende } \\
\text { Nachbarn }(G W, 04.11 .2014) \\
\text { Schwulen }(G W, 04.11 .2014) \\
\text { die Älteren }(G W, 04.11 .2014) \\
\text { die Kranken }(G W, 06.03 .2015)\end{array}$ & $\begin{array}{l}\text { anders Liebende (Spiegel, 12.02.2014) } \\
\text { die Folter (Spiegel, 04.09.2014) } \\
\text { behinderte Politiker (Spiegel, 03.11.2014) }\end{array}$ \\
\hline \multicolumn{2}{|c|}{ Taten } \\
\hline $\begin{array}{l}\text { Korruption }(G W, 27.03 .2015) \\
\text { Pädophilie }(N e w s w e e k, 09.10 .2013) \\
\text { unangebrachtes Verhalten }(N e w s w e e k, \\
13.03 .2015) \\
\text { Abtreibung }(R z, 13.02 .2015) \\
\text { Verkehrsvergehen }(\text { Newsweek, } \\
22.01 .2015) \\
\text { Überschreitung der Geschwindigkeit }(R z \text {, } \\
03.01 .2013) \\
\text { Gewalt }(R z, 29.09 .2014) \\
\text { Plagiat }(G W, 11.10 .2013) \\
\text { Pyrotechnik }(R z, 05.01 .2015) \\
\text { Zufluchten für Terroristen }(R z, \\
\text { 23.09.2014) } \\
\text { Partys am Samstag }(R z, 10.11 .2014) \\
\text { Angriffe der Demonstranten }(\text { Newsweek, } \\
\text { 30.11.2013) }\end{array}$ & $\begin{array}{l}\text { Korruption (Spiegel, 03.12.2014) } \\
\text { Pädophilie (Spiegel, 02.09.2013) } \\
\text { Verletzung der ethischen Regeln (Spiegel, } \\
\text { 02.10.2014) } \\
\text { Verhaltensweise (Spiegel, 18.08.2014) } \\
\text { sexueller Missbrauch (Spiegel, 02.09.2013) } \\
\text { Verschleierung des Gesichts (F.A.Z., } \\
\text { 21.01.2015 (c)) } \\
\text { Protest (Spiegel, 15.01.2015) }\end{array}$ \\
\hline \multicolumn{2}{|c|}{ Ansichten } \\
\hline $\begin{array}{l}\text { andere politische Gesinnung }(G W, \\
04.11 .2014) \\
\text { andere Religion }(G W, 04.11 .2014) \\
\text { sexuelle Orientierung }(R z, 28.01 .2015)\end{array}$ & $\begin{array}{l}\text { andere Kulturen }(S Z, 13.02 .2015) \text { Religio- } \\
\text { nen }(S Z, 13.02 .2015) \\
\text { andere Glaubensrichtungen (Spiegel, } \\
20.11 .2014) \\
\text { homosexuelle Lebensentwürfe (Spiegel, } \\
10.02 .2014)\end{array}$ \\
\hline \multicolumn{2}{|c|}{ Stoffe/Substanzen } \\
\hline $\begin{array}{l}\text { Glukose }(R z, 04.03 .2014 ; \text { Newsweek, } \\
14.01 .2015)\end{array}$ & $\begin{array}{l}\text { Futtermittel (Spiegel, 13.12.2013) } \\
\text { Eiweiße (Spiegel, 20.12.2013) } \\
\text { genmanipulierte Stoffe (Spiegel, } \\
13.12 .2013)\end{array}$ \\
\hline
\end{tabular}

${ }^{10}$ In Bezug auf nicht heterosexuelle Personen. 


\begin{tabular}{|c|c|}
\hline & $\begin{array}{l}\text { Myelin (Spiegel, 20.12.2013) } \\
\text { Alkohol (SZ, 29.01.2015; SZ, 28.01.205) }\end{array}$ \\
\hline \multicolumn{2}{|r|}{ Aussehen } \\
\hline andere Hautfarbe $(G W, 04.11 .2014)$ & Aussehen (Spiegel, 18.08.2014) \\
\hline \multirow{2}{*}{\multicolumn{2}{|c|}{ 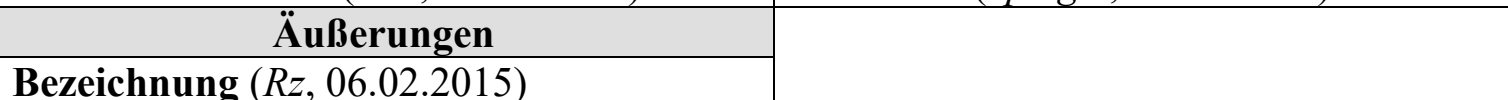 }} \\
\hline & \\
\hline \multirow{2}{*}{\multicolumn{2}{|c|}{$\begin{array}{c}\text { Herkunft } \\
\text { andere Nationalität }(G W, 04.11 .2014)\end{array}$}} \\
\hline & \\
\hline \multirow{2}{*}{\multicolumn{2}{|c|}{$\begin{array}{c}\text { Werbung } \\
\text { Werbungen }(G W, 23.09 .2014) \\
\end{array}$}} \\
\hline & \\
\hline & Krankheiten/körperliche Reaktionen \\
\hline & $\begin{array}{l}\text { psychische Erkrankungen (Spiegel, } \\
\text { 28.03.2015) } \\
\text { Behinderung der Politiker (Spiegel, } \\
\text { 03.11.2014) } \\
\text { Entzündungsreaktionen (Spiegel, } \\
\text { 21.10.2014) } \\
\text { Darmschädigungen (Spiegel, 21.10.2014) }\end{array}$ \\
\hline & Eigenschaften \\
\hline & $\begin{array}{l}\text { Ambivalenz (Spiegel, 29.12.2014) } \\
\text { Eigenheit (Spiegel, 29.12.2014) }\end{array}$ \\
\hline & Tiere \\
\hline & Raubtiere (Spiegel, 10.01.2014 (a)) \\
\hline & Währung \\
\hline & starker Dollar (F.A.Z., 21.01.2015 (a)) \\
\hline & Behandlungsmethode \\
\hline & Therapie (Spiegel, 27.03.2014) \\
\hline & Medien \\
\hline & Medien (Spiegel, 15.01.2015) \\
\hline
\end{tabular}

Tabelle 4: Konkrete Füllwerte der Leerstelle „Wer oder was kann (nicht) toleriert werden?““ auf der Basis von den polnischen und den deutschen Presseartikeln.

Die in der dritten Tabelle angegebenen Daten betreffen die Frage, wo man es mit der Toleranz zu tun hat. Es wurden insgesamt sieben polnische und acht deutsche konkrete Füllwerte aufgelistet:

\section{Wo findet (keine) Toleranz statt?}

im Straßenverkehr (Newsweek,

22.01.2015; $R z, 03.01 .2013)$

während des Marsches $(R z, 05.01 .2015)$

in der Gesellschaft (Newsweek, 13.03.2015)

in der Welt $(G W, 06.03 .2015)$

in der Kirche (Newsweek, 09.10.2013)

im Nordkorea (Newsweek, 04.08.2015)

im Internet (Newsweek, 01.08.2015) im Straßenverkehr $(S Z, 28.01 .2015)$

im Schulunterricht (Spiegel, 10.02.2014)

in Justiz (Spiegel, 04.09.2014)

in Polizei (Spiegel, 04.09.2014)

in Militär (Spiegel, 04.09.2014)

auf dem Weg (Spiegel, 10.05.2013)

in der EU (Spiegel, 13.12.2013)

Tabelle 5: Konkrete Füllwerte der Leerstelle „Wo findet (keine) Toleranz statt?“ auf der Basis von den polnischen und den deutschen Presseartikeln.

Die vierte Tabelle schildert die Eigenschaften von Toleranz. In allen untersuchten Artikeln wurden fünf polnische und acht deutsche konkrete Füllwerte notiert. Wie aus der Tabelle re- 
sultiert, bezieht sich die Mehrheit der konkreten Füllwerte auf dieselbe Eigenschaft, d. h. Fähigkeit der Toleranz zur Vergrößerung oder Verringerung ihres Grades, aber in gewissen Grenzen.

\begin{tabular}{|l|l|}
\hline \multicolumn{2}{|c|}{ Durch welche Eigenschaften kennzeichnet sich Toleranz? } \\
\hline hat die Grenzen $(G W, 04.11 .2014)$ & hat die Grenzen (Spiegel, 02.09.2013) \\
kann erweitert werden $($ Newsweek, & ist nicht einfach $($ SZ, 15.01.2015) \\
$01.08 .2015)$ & lässt sich erlernen $(S Z, 15.01 .2015)$ \\
kann untersucht werden $(G W$, & kann wachsen (Spiegel, 03.11.2014) \\
$04.11 .2014)$ & lässt sich steigern (Spiegel, 05.08.2013) \\
kann gestört sein11 $(R z, 04.03 .2014 ;$ & kann erhöht werden12 (Spiegel, 20.12.2013) \\
Newsweek, 14.01.2015) & kann abnehmen (Spiegel, 15.01.2015) \\
hat die jahrhundertealte Tradition & lässt sich einüben (Spiegel, 18.08.2014) \\
(Newsweek, 02.10.2015) & kann hervorgerufen werden (Spiegel, \\
& 20.12.2013) \\
& kann variieren (Spiegel, 10.05.2013) \\
\hline
\end{tabular}

Tabelle 6: Konkrete Füllwerte der Leerstelle „Durch welche Eigenschaften kennzeichnet sich Toleranz?“ auf der Basis von den polnischen und den deutschen Presseartikeln.

In der letzten Tabelle wurden synonymische Bezeichnungen für Toleranz dargelegt. In allen polnischen Pressetexten wurde lediglich ein Synonym gefunden, in deutschen Presseartikeln gab es nur zwei synonymische Ausdrücke.

\begin{tabular}{|l|l|}
\hline \multicolumn{2}{|c|}{ Welche anderen Bezeichnungen beziehen sich auf Toleranz? } \\
\hline Ertragen (Newsweek, 23.09.2014) & $\begin{array}{l}\text { Duldung (Spiegel, 12.02.2014) } \\
\text { Resistenz (Spiegel, 05.08.2013) }\end{array}$ \\
\hline
\end{tabular}

Tabelle 7: Konkrete Füllwerte der Leerstelle „Welche Bezeichnungen beziehen sich auf Toleranz?“" auf der Basis von den polnischen und den deutschen Presseartikeln.

Die unten präsentierte Abbildung zeigt, wie viele Füllwerte den einzelnen Leerstellen zugeordnet wurden. Vergleicht man die Prozentzahlen, stellt man fest, dass die zwei ersten Leerstellen sowohl im Polnischen als auch im Deutschen besonders auffällig sind. Nach Ziem (2008: 342) zeugt dies von einer Verfestigung dieser Leerstellen im Vergleich zu den anderen.

\footnotetext{
11 In Bezug auf Glukosetoleranz.

12 Immuntoleranz.
} 


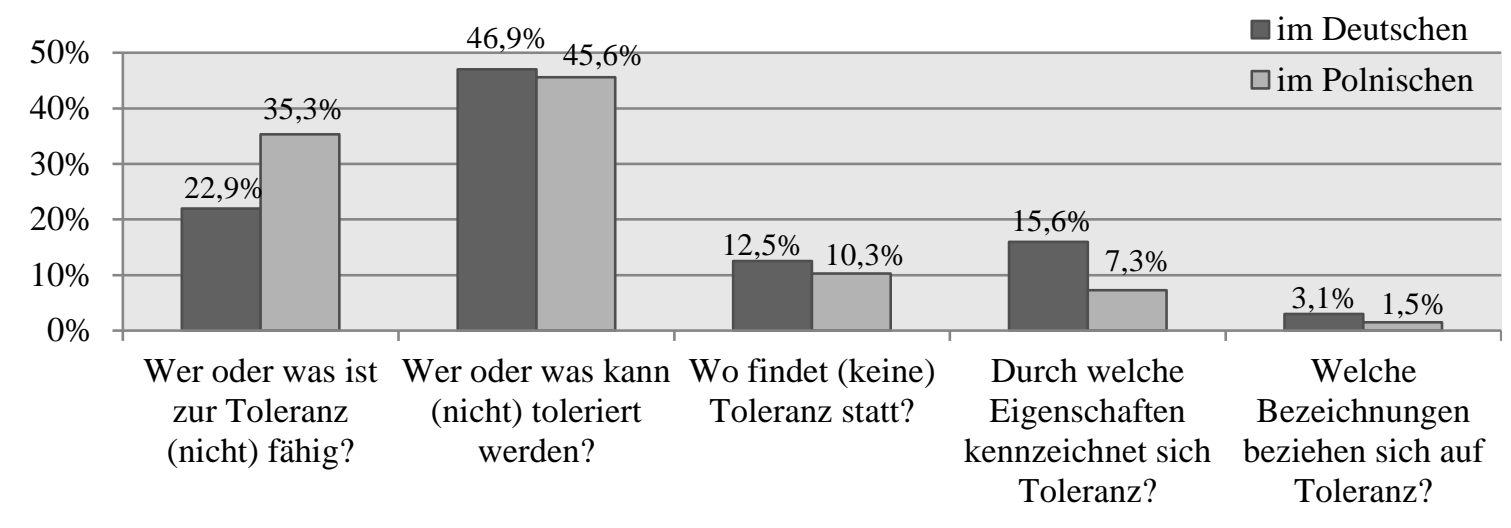

Abbildung 3: Prozentualer Anteil konkreter Füllwerte an den einzelnen Leerstellen des Frames TOLERANZ im Deutschen und im Polnischen.

\section{$5 \quad$ Vergleichende Bemerkungen}

Nach der Analyse aller angesammelten Korpusdaten und aus dem Vergleich zwischen der polnischen und der deutschen kognitiven Definition von Toleranz kommen einige bemerkenswerte Schlussfolgerungen zum Vorschein. Sie werden an dieser Stelle stichwortartig zusammengefasst:

1. Die Definitionen von Toleranz unterscheiden sich im Deutschen und im Polnischen in der Länge und Anzahl der angegebenen Bedeutungsvarianten. Die polnische lexikographische Definition enthält acht Bedeutungsvarianten, wobei drei Varianten „eine von Juden entrichtete Steuer, die sie vom Militärdienst befreite“, „Fähigkeit der Fotoemulsion zum Ausgleichen der Belichtungsfehler“ und „Anerkennung jedes Menschen für einen möglichen Partner des Dialogs“" in den deutschen Wörterbüchern nicht vorkommen. In den deutschen Wörterbüchern werden insgesamt fünf Bedeutungsvarianten von Toleranz notiert, wobei die Bestimmung von Toleranz als „Wirkungsabfall des Medikamentes als Antwort des Körpers nach wiederholter Gabe“ im Polnischen fehlt.

2. Für den Frame TOLERANZ im Deutschen und im Polnischen werden mithilfe der schriftlichen Befragung zum Teil andere Standardwerte festgestellt. Im Polnischen handelt es sich um Toleranz als „Respekt vor anderen religiösen Anschauungen“, „einer anderen politischen Gesinnung“ und ,einer anderen sexuellen Orientierung“. Für die deutschen MuttersprachlerInnen sind die Bedeutungsvarianten ,zulässige Abweichung von einem geforderten Wert, z. B. Länge“, „Respekt vor einer anderen sexuellen Orientierung“, „Respekt vor einer anderen Religion“ und „Respekt vor einer anderen politischen Gesinnung“ Standardwerte des Frames TOLERANZ.

3. Die Befragung unter den polnischen MuttersprachlerInnen zeigte, dass sie achten, akzeptieren und verständnisvoll sein für Synonyme des Verbes tolerieren halten. Im Deutschen wurde dagegen nur ein synonymischer Ausdruck (verständnisvoll sein) als Standardwert des Frames TOLERANZ festgestellt.

An dieser Stelle muss betont werden, dass die Analyse keinen Anspruch auf Vollständigkeit hinsichtlich der Bestimmung der Standardwerte in Bezug auf die ganze Population erhebt. Die Anzahl von UmfrageteilnehmerInnen ist zu gering, als dass man die 
Ergebnisse verallgemeinern könnte, und gleichzeitig ausreichend, um festzustellen, dass der Begriff unterschiedlich konzeptualisiert werden kann. Davon zeugen die Ergebnisse aus der Stichprobe, nämlich die beachtlichen Differenzen in Prozentwerten. Die Auswertung der Ergebnisse macht deutlich, dass sich bestimmte Bedeutungsvarianten von Toleranz (in Bezug auf die Stichprobe) im Vergleich mit den anderen durchgesetzt haben.

4. Viele Informationen über die aktuelle Anwendung des Wortes „Toleranz“, die in den Wörterbüchern nicht enthalten waren, lieferte die Analyse der deutschen und polnischen Pressetexte. Daraus ergibt sich eine große Anzahl von konkreten Füllwerten, die in den Wörterbüchern fehlten. Die ausführlichen Ergebnisse wurden schon im vorigen Kapitel präsentiert. An dieser Stelle wird auf die bemerkenswerten Beispiele aus der Presse (separat für jede Leerstelle) aufmerksam gemacht.

(1) „Wer oder was ist zur Toleranz (nicht) fähig?“

Sowohl in den deutschen als in den polnischen Wörterbüchern sind meistens Menschen/Organismen zur Toleranz fähig. In der Presse kommen noch andere Agens vor, z. B.

- Institutionen: Offenbar werden die Zentralbanker einen starken Dollar tolerieren. (F.A.Z., 21.01.2015 (a))

- Systeme: Und falls doch, gibt es als kleinen Kompromiss eine spezielle Traktionskontrolle, deren Toleranz je nach Untergrund variiert werden kann. (Spiegel, 10.05.2013)

- Land: Schweden ist für seine Toleranz gegenüber der sexuellen Orientierung bekannt. $(R z, 28.01 .2015)$

(2) „Wen oder was kann man (nicht) tolerieren?““

Die Wörterbücher berücksichtigen zwar viele Patiens/Betroffene (z. B. Anschauungen, Religion, Temperatur oder Medikamente), die Pressetexte erweitern diese Liste erheblich:

- Pädophilie: Wir betonen, dass es keine Toleranz für Pädophilie gibt. (Newsweek, 09.10.2013)

- körperliche Reaktionen: (...) durch ihre Eigenschaft als Superverbreiter auch eine Toleranz gegenüber den antibiotisch begründeten Darmschädigungen und Entzündungsreaktionen haben. (Spiegel, 21.10.2014)

- Behandlungsmethode: Patienten, die während einer Chemotherapie Sport treiben, tolerieren die Therapie besser. (Spiegel, 27.03.2014)

\section{(3) „Wo findet (keine) Toleranz statt?“}

Die Wörterbücher beschränken sich auf die Angabe unterschiedlicher Bereiche, in denen der Begriff „Toleranz“ benutzt wird, z. B. Toleranz in der Medizin, im religiösen, politischen oder technischen Bereich. In den Presseartikeln kommen dagegen viele explizite Füllwerte vor, z. B.

- im Straßenverkehr: Eine Null-Toleranz-Grenze für alle im Straßenverkehr. Autos, Motorräder, Roller, Fahrräder. Alkohol hat dort nichts zu suchen. Wer trinken will, lässt sich fahren. Oder geht zu Fuß. (SZ 28.01.2015) 
- in der Kirche: Wir betonen - es gibt keine Toleranz für Pädophilie. Diesen Standpunkt vertritt die ganze Kirche in Polen - sowohl die Geistlichen als auch die katholischen Laien. (Newsweek, 09.10.2013)

- $\quad$ in Justiz, Polizei und Militär: Polizei oder Militär erpressen mit Gewalt Geständnisse von Unschuldigen und machen sie so zu "presuntos culpables", zu "mutmaßlich Schuldigen". In Justiz, Polizei und Militär herrsche Toleranz gegenüber Folter, stellt Amnesty fest. Die Täter gehen fast immer straffrei aus. (Spiegel, 04.09.2014)

(4) „Durch welche Eigenschaften kennzeichnet sich Toleranz?“

Im Vergleich zu den Wörterbüchern sind die Pressetexte an den Füllwerten reicher, die sich auf die Eigenschaften der Toleranz beziehen. Das Merkmal ,hat die Grenzen' steht in den Wörterbüchern meistens unter dem Stichwort Toleranzgrenze. Es lassen sich folgende Eigenschaften der Toleranz darstellen, die in den Presseartikeln explizit genannt werden:

- Kann wachsen: Andererseits scheint es eine wachsende Toleranz für Politiker mit Behinderungen zu geben. (Spiegel, 03.11.2014)

- lässt sich erlernen: Ein tolerantes Land schützt die Satire als nicht nur witzigen, sondern auch wertvollen Beitrag zum Meinungskampf. Toleranz ist nicht einfach, aber sie lässt sich erlernen. (SZ, 15.01.2015)

- kann gestört sein: „,...) 33,5 Millionen Europäer haben eine gestörte Glukosetoleranz, die zum Diabetes führt. “ $(R z, 04.2014)$

(5) „Welche anderen Bezeichnungen beziehen sich auf Toleranz?“

Die Wörterbücher lieferten mehr synonymische Ausdrücke für Toleranz. In den insgesamt 60 analysierten Pressetexten gibt es nur drei Synonyme, wobei all diese Ausdrücke auch in den Wörterbüchern vorkommen.

- Ertragen: „Gehen wir von der Toleranz aus, derer Begriff aus dem Lateinischen einzig und allein ,Ertragen“ bedeutet (...)“ (Newsweek, 23.09.2014)

- Duldung: „Während nämlich die bloße Toleranz, also die schlichte Duldung der anders Liebenden, völlig unproblematisch sei, bedeute Akzeptanz eine Zustimmung. “ (Spiegel 12.02.2014)

- Resistenz: Die Toleranz gegenüber Dürre ließe sich mit gentechnischen Methoden möglicherweise noch weiter steigern, meint Yusaku Uga. Er halte das für eine von mehreren sinnvollen Strategien, die Dürreresistenz von Reis zu steigern. (Spiegel 05.08.2013).

\section{Ausblick}

Die frame-semantische Perspektive ist bei der Darstellung einer kognitiven Definition eines sprachlichen Ausdrucks von großem Vorteil, weil sie das Postulat der Verstehensrelevanz realisiert und alle Bedeutungsaspekte im Unterschied zu Definitionen, die mittels des modularistischen Ansatzes formuliert wurden, berücksichtigt. Die Einteilung der Prädikate in konkrete Füllwerte und Standardwerte bringt darüber hinaus zum Vorschein, dass die einzelnen Be- 
deutungsvarianten für die SprachbenutzerInnen einen unterschiedlichen Stellenwert aufweisen.

Wenn es sich um die Darstellung des Begriffs „Toleranz“ im Deutschen und im Polnischen handelt, bleiben einige Aspekte unerforscht:

1. Welche Faktoren beeinflussten die Ausdifferenzierung der polnischen und der deutschen Definition von Toleranz?

2. Welche Prädikate, die in den polnischen und deutschen Presseartikeln auftauchen, gehören zu den konkreten Füllwerten und welche Prädikate kann man für Standardwerte des Frames TOLERANZ erklären?

Die Analyse des Begriffs „Toleranz“ in Anlehnung an ein umfangreicheres Untersuchungskorpus und eine noch mehr ausgebaute Befragung könnte - wessen sich die Autorin bewusst ist - eine Ergänzung der dargestellten Ergebnisse liefern und die bisher unerforschten Aspekte erklären. Sogar das in dem Beitrag präsentierte Korpus ermöglicht, die Unterschiede zwischen der Definition von Toleranz im Deutschen und im Polnischen zu veranschaulichen, und lässt als empirische Grundlage das von der Autorin vorgeschlagene Verfahren als ergiebig erscheinen.

\section{Literaturverzeichnis}

Barsalou, Lawrence W. (1992): "Frames, Concepts, and Conceptual Fields". In: Kittay, Eva Feder/Lehrer, Adrienne (eds.): Frames, Fields, and Contrast. New Essays in Semantic and Lexical Organisation. Hillsdale (New Jersey), Lawrence Erlbaum Associates: 21-74.

Bierwisch, Manfred (1983): „Semantische und konzeptuelle Repräsentation lexikalischer Einheiten“. In Růžička, Rudolf/Motsch, Wolfgang (eds.): Untersuchungen zur Semantik. Berlin, Akademie-Verlag: 61-101.

Busse, Dietrich (2012): Frame-Semantik: Ein Kompendium. Berlin: de Gruyter.

Charniak, Eugen (1977): "A Framed Painting. The Representation of a Common Sense Knowledge Fragment”. Cognitive Science 1/4: 235-264.

Czachur, Waldemar (2011): Diskursive Weltbilder im Kontrast. Linguistische Konzeptionen und Methode der kontrastiven Diskursanalyse deutscher und polnischer Medien. Wrocław: Oficyna Wydawnicza ATUT.

Dereń, Ewa/Polański, Edward (2008): Wielki słownik języka polskiego. Kraków: Krakowskie Wydawnictwo Naukowe.

Długosz-Kurczabowa, Krystyna (2005): Słownik etymologiczny języka polskiego. Warszawa: Wydawnictwo Naukowe PWN.

Dubisz, Stanisław (2003): Uniwersalny słownik języka polskiego. Warszawa: Wydawnictwo Naukowe PWN.

Duden (2002): Deutsches Universalwörterbuch. Mannheim: Dudenverlag.

Duden (1989): Etymologie. Herkunftswörterbuch der deutschen Sprache. Mannheim: Dudenverlag.

Fillmore, Charles J. (1975): “An Alternative to Checklist Theories of Meaning". In: Cogen, Cathy et al. (eds.): Proceeding of the First Annual Meeting of the Berkeley Linguistics Society. Berkeley, The Society: 123-131. 
Konerding, Klaus-Peter (1993): Frames und lexikalisches Bedeutungswissen. Untersuchungen zur linguistischen Grundlegung einer Frametheorie und zu ihrer Anwendung in der Lexikographie. Tübingen: Niemeyer.

Minsky, Marvin (1975): “A Framework for Representing Knowledge”. In: Winston, Patrick Henry (ed.): The Psychology of Computer Vision. New York etc., McGraw-Hill: 211-277.

Paul, Hermann (2002): Deutsches Wörterbuch. Bedeutungsgeschichte und Aufbau unseres Wortschatzes. Tübingen: Niemeyer.

Petersen, Wiebke (2007): „Representation of Concepts as Frames“. The Baltic International Yearbook of Cognition, Logic and Communication 2: 151-170.

Schank, Roger C./Abelson, Robert P. (1977): Scripts, Plans, Goals and Understanding: An Inquiry into Human Knowledge Structures. Hillsdale (Michigan): Lawrence Erlbaum Associates.

Schwarz, Monika (1992): Einführung in die kognitive Linguistik. Tübingen: Francke.

Schwarz, Monika (2002): „Konzeptuelle Ansätze. Einebenen-Ansatz vs. Mehrebenenansatz“. In: Cruse, David Alan et al. (eds.): Lexikologie / Lexicology. Ein internationales Handbuch zur Natur und Struktur von Wörtern und Wortschätzen. Berlin, de Gruyter: 277-284 (= Handbücher zu Sprach- und Kommunikationswissenschaft 21).

Strube, Gerhard et al. (eds.) (1996): Wörterbuch der Kognitionswissenschaft. Stuttgart: KlettCotta.

Zgółkowa, Halina (2003): Praktyczny słownik współczesnej polszczyzny. Poznań: Wydawnictwo „Kurpisz“.

Ziem, Alexander (2008): Frames und sprachliches Wissen. Kognitive Aspekte der semantischen Kompetenz. Berlin/New York: de Gruyter (= Sprache und Wissen 2).

\section{Internetquellen:}

[ohne Autorenangabe] (2015): "Film o Putinie: Prezydent-cudotwórca. 15 lat rosyjskiej propagandy w pigułce”. Newsweek Polska 27.04.15. http://swiat.newsweek.pl/film-oprezydenturze-wladimira-putina,artykuly,362020,1.html [12.12.2015].

[ohne Autorenangabe] (2015): “Jedna czwarta brytyjskich muzułmanów sympatyzuje z przyczynami ataków na 'Charlie Hebdo"'. Newsweek Polska 25.02.15. http://swiat.newsweek.pl/brytyjscy-muzulmanie-sympatyzuja-z-motywami-atakow-nacharlie-hebdo,artykuly,357852,1.html [12.12.2015].

[ohne Autorenangabe] (2015): “Jest pierwszy pozew Durczoka przeciwko 'Wprost"”. Newsweek Polska 13.03.15. http://polska.newsweek.pl/sprawa-kamila-durczoka-w-tvndochodzilo-do-mobbingu-i-molestowania-, artykuly,358690,1.html [14.03.2015].

[ohne Autorenangabe] (2015): "Korea Płn. 'najbardziej wrogim chrześcijanom krajem świata”. Newsweek Polska 04.08.15. http://swiat.newsweek.pl/korea-pln-najbardziejwrogim-chrzescijanom-krajem-swiata-,artykuly,368053,1.html [12.12.2015].

[ohne Autorenangabe] (2015): "Naukowcy: Homofob to często ukryty gej”. Newsweek Polska 06.07.15. http://swiat.newsweek.pl/homofobi-ukrycihomsesualisci,artykuly,366350,1.html [12.12.2015].

[ohne Autorenangabe] (2015): "Polski ksiądz w Rzymie: Jestem gejem. Watykan: Musi opuścić miejsce pracy”. Newsweek Polska 02.10.15. http://swiat.newsweek.pl/krzysztofcharamsa-polski-ksiadz-jestem-gejem,artykuly,371554,1.html [11.12.2015]. 
[ohne Autorenangabe] (2015): "Sondaż: Polacy bezwzględni dla pijanych kierowców, ale nie dla piratów drogowych". Newsweek Polska 22.01.15. http://polska.newsweek.pl/polacybezwzgledni-dla-pijanych-kierowcow-chca-surowych-kar,artykuly,355610,1.html [02.02.2015].

[ohne Autorenangabe] (2015): "W Polsce mamy coraz więcej rasizmu. Przystanek Woodstock z tym walczy". Newsweeks Polska 01.08.15. http://polska.newsweek.pl/woodstockprzeciwko-rasizmowi-stowarzyszenie-nigdy-wiecej,artykuly,367834,1.html [12.12.2015].

[ohne Autorenangabe] (2014): „'Puls Biznesu': Dobrana reklama bardziej tolerowana”. Wyborcza.

http://wyborcza.pl/1,91446,16688784,_Puls_Biznesu__Dobrana_reklama_bardziej_tolero wana.html?pelna=tak [15.01.2015].

[ohne Autorenangabe] (2014): "Tomasz Lis na żywo: Dariusz Michalczewski vs Beata Kempa. Bitwa o tolerancję". Newsweek Polska 23.09.14. http://polska.newsweek.pl/tomasz-lis-na-zywo-dariusz-michalczewski-vs-beatakempa,artykuly,348239,1.html_[02.02.2015].

[ohne Autorenangabe] (2013): "Donald Tusk o Ukrainie: Nie będziemy tego tolerować". Newsweek Polska 30.11.13. http://swiat.newsweek.pl/donald-tusk-o-sytuacji-na-ukrainiemajdan-niepodleglosci-newsweek-pl,artykuly,275948,1.html [15.01.2015].

[ohne Autorenangabe] (2013): "Kościół mówi pedofilii stanowcze nie". Newsweek Polska 09.10.13. http://polska.newsweek.pl/kosciol-pedofilia-sprzeciw-pedofilii-bp-polaknewsweek-pl,artykuly,272191,1.html [15.01.2015].

[ohne Autorenangabe] (2013): "Soczi. Tęczowe mimo represji”. Newsweek Polska 19.12.13. http://swiat.newsweek.pl/zakaz-promocji-homoseksualizmu-w-rosji-a-igrzyska-w-soczinewsweek-pl,galeria,277328,1.html_[02.02.2015].

[ohne Autorenangabe] (2013): "Tuberkulose-Impfstoff bremst möglicherweise Multiple Sklerose“. Spiegel Online 20.12.13. http://www.spiegel.de/gesundheit/diagnose/impfstoffgegen-ms-neue-therapieversuche-mit-tbc-medikament-a-937392.html [15.01.2015].

Ahrens, Peter (2014): „Auch der zweite Iglinski-Bruder erwischt“. Spiegel Online 02.10.14. http://www.spiegel.de/sport/sonst/radsport-maxim-iglinski-von-astana-wegen-dopingerwischt-a-995051.html [15.01.2015].

Becker, Markus (2015): "Verdrängen, verleugnen, verschweigen”. Spiegel Online 28.03.15. http://www.spiegel.de/wissenschaft/mensch/germanwings-psychische-probleme-werdenin-der-branche-verdraengt-a-1025983.html [28.03.2015].

Bielecki, Jędrzej (2015): “Aborcja łatwa jak pocałunek”. Rzeczpospolita 14.02.15. http://www.rp.pl/artykul/1178813-Aborcja-latwa-jak-pocalunek.html [14.02.2015].

Błaszczak, Grażyna (2014): “Czynsz zamiast raty”. Rzeczpospolita 10.11.14. http://www.rp.pl/artykul/1156049-Czynsz-zamiast-raty.html [02.02.2015].

Diekmann, Florian (2013): "Was das Amflora-Verbot für Verbraucher bedeutet". Spiegel Online 13.12.13. http://www.spiegel.de/wirtschaft/service/gentechnik-kartoffel-amflora-wasdas-verbot-per-urteil-bedeutet-a-938976.html [15.01.2015].

Dpa (2015): "Schwere Kämpfe am Flughafen von Donezk". Frankfurter Allgemeine 18.01.15. http://www.faz.net/aktuell/politik/ukraine-schwere-kaempfe-am-flughafen-von-donezk13377362.html [02.03.2015]. 
Dpa-AFX/Reuters (2015): "Gewinne an der Wall Street schieben deutsche Aktien an" Frankfurter Allgemeine 18.01.15. http://www.faz.net/aktuell/finanzen/aktien/marktberichtgewinne-an-der-wall-street-schieben-deutsche-aktien-an-13359120.html [02.03.2015].

Dürmeier, Franziska (2015): „Auf dem Weg zur Annäherung“. Süddeutsche Zeitung 13.02.15. http://www.sueddeutsche.de/muenchen/landkreismuenchen/islam-debatte-auf-dem-wegzur-annaeherung-1.2350503 [13.03.2015].

Ehringfeld, Klaus (2014): "Dein Feind und Folterer". Spiegel Online 04.09.14. http://www.spiegel.de/panorama/justiz/folter-in-mexiko-polizeigewalt-wird-kaumgeahndet-a-989821.html [15.01.2015].

Elmer, Christina (2014): „Warum Ökosysteme Löwen und Luchse brauchen“. Spiegel Online 10.01.14. http://www.spiegel.de/wissenschaft/natur/raubtiere-warum-oekosysteme-loewenund-luchse-brauchen-a-942665.html [15.01.2015].

Fleischhauer, Jan/Friedmann, Jan (2014): “'Am Rand zu stehen ist schrecklich”". Der Spiegel 14/34. http://www.spiegel.de/spiegel/print/d-128743710.html [15.01.2015].

Gajos-Kaniewska, Dorota (2013): "Fotoradary bardziej tolerancyjne dla kierowcow". Rzeczpospolita 03.01.13. http://prawo.rp.pl/artykul/966182.html [02.02.2015].

Gathmann, Florian et al. (2013): “'Das Tabu durchgebrochen"”. Der Spiegel 13/36. http://www.spiegel.de/spiegel/print/d-110117926.html [15.01.2015].

Gensch, Valentin (2014): “AAls ich im Boot saß, spürte ich die Übelkeit nicht mehr““. Spiegel Online 27.03.14. http://www.spiegel.de/gesundheit/diagnose/rudern-gegen-krebs-wiesport-waehrend-der-chemo-helfen-kann-a-960837.html [15.01.2015].

Grünweg, Tom (2013): „Der Kleine für große Gefühle“. Spiegel Online 10.05.13. http://www.spiegel.de/auto/fahrberichte/peugeot-2008-der-hoeher-gelegte-kleinwagen-istein-agiles-stadtauto-a-898605.html [15.01.2015].

Grünweg, Tom (2015): “Alle Räder greifen zu”. Spiegel Online 10.02.15. http://www.spiegel.de/auto/fahrberichte/jaguar-f-type-awd-fahrbericht-sportwagen-jetztmit-allradantrieb-a-1016435.html [16.02.2015].

Harloff, Thomas (2015): „Das müssen Sie über Promillegrenzen wissen“. Süddeutsche Zeitung 29.01.15. http://www.sueddeutsche.de/auto/zum-verkehrsgerichtstag-das-muessensie-ueber-promillegrenzen-wissen-1.2325110 [10.02.2015].

Haszczyński, Jerzy (2015): "Pocieszające wieści z Niemiec w sprawie ,polskich obozów"”. Rzeczpospolita 06.02.15. http://www.rp.pl/artykul/1177524-Pocieszajace-wiesci-zNiemiec-w-sprawie--polskich-obozow-.html [07.02.2015].

Hoffmann, Christiane/Pfister René (2014): „'Wort, Pause, Stottern, Wort, Pause““. Der Spiegel 45/14. http://www.spiegel.de/spiegel/print/d-130092973.html [15.01.2015].

Köpf, Matthias (2015): „Die Mission des Diakons“. Süddeutsche Zeitung 04.02.15. http://www.sueddeutsche.de/muenchen/wolfratshausen/kreuze-in-klassenzimmern-diemission-des-diakons-1.2334690 [10.02.2015].

Kowalska, Karolina (2014): „Europy nie stać na cukrzycę”. Rzeczpospolita 04.03.14. http://www.rp.pl/artykul/1091470-Europy-nie-stac-na-cukrzyce.html [02.02.2015].

Krężlewicz-Dzieciątek, Małgorzata (2014): "Strażnicy podejrzani o znęcanie się nad uchodźcami w ośrodku dla azylantów w Niemczech". Rzeczpospolita 29.09.14. http://www.rp.pl/artykul/1144827-Straznicy-podejrzani-o-znecanie-sie-nad-uchodzcamiw-osrodku-dla-azylantow-w-Niemczech.html [02.02.2015]. 
Kuzmany, Stefan (2014): "Wenn Worte wehtun". Spiegel Online 12.02.14. http://www.spiegel.de/kultur/tv/tv-kritik-zu-maischberger-talk-ueber-homosexualitaet-a952888.html [15.01.2015].

Malangré, Claudia (2014): „'Unangebrachte Witze sind Alltag'”. Spiegel Online 19.09.14. http://www.spiegel.de/schulspiegel/wissen/der-vorsitzende-des-landesschuelerratesniedersachsen-ueber-sexuelle-vielfalt-im-unterricht-a-992387.html [15.01.2015].

Malinowski, Przemysław (2014): “Obama: USA nie walczą z Państwem Islamskim same”. Rzeczpospolita 23.09.14. http://www.rp.pl/artykul/1143517-Obama--USA-nie-walcza-zPanstwem-Islamskim-same.html [02.02.2015].

Marquart, Maria (2014): „Mit dem Aufstieg fließt das Schmiergeld“. Spiegel Online 03.12.14. http://www.spiegel.de/wirtschaft/soziales/korruption-transparency-ranking-prangert-chinaund-tuerkei-an-a-1006310.html [15.01.2015].

Merlot, Julia (2014): „Antibiotika verfehlen Wirkung gegen Superverbreiter“. Spiegel Online 21.10.14. http://www.spiegel.de/wissenschaft/medizin/superverbreiter-reagieren-nicht-aufantibiotika-a-998246.html [15.01.2015].

Michalak, Ada (2015): "Policja żąda 5 tys. zł grzywny dla szefa Marszu Niepodległości'13”. Rzeczpospolita 05.01.15. http://www.rp.pl/artykul/1168992-Policja-zada-5-tys--zlgrzywny-dla-szefa-Marszu-Niepodleglosci-13.html [02.02.2015].

Michalak, Ada (2015): "Szwecja: "Neutralna płciowo" przebieralnia na basenie". Rzeczpospolita 28.01.15. http://www.rp.pl/artykul/1175147-Szwecja---Neutralna-plciowo-przebieralnia-na-basenie.html [02.02.2015].

Milewski Piotr (2013): “Ameryka über alles. Kraj uprzedzeń i nietolerancji”. Newsweek Polska 06.10.13. http://swiat.newsweek.pl/stany-zjednoczone-krajem-uprzedzen-inietolerancji-na-newsweek-pl,artykuly,270851,1.html [15.01.2015].

Padtberg, Carola (2014): „Sorry, diese Schule ist zu weiß”. Spiegel Online 20.11.14. http://www.spiegel.de/schulspiegel/schule-in-grossbritannien-in-der-kritik-zu-vieleweisse-schueler-a-1004014.html [15.01.2015].

Pawłowska, Anna (2014): "Wolimy mieszkać koło prostytutki niż koło członka sekty. CBOS zbadał granice tolerancji Polaków". Wyborcza. http://wborcza.pl/1,75478,16913577,Wolimy_miezkac_kolo_prostytutki_niz_kolo_czlonk a.html [15.01.2015].

Reek, Felix (2015): „Die Vernunft schwindet mit jedem Schluck“. Süddeutsche Zeitung 28.01.15. http://www.sueddeutsche.de/auto/alkohol-beim-radfahren-die-vernunftschwindet-mit-jedem-schluck-1.2322388 [10.02.2015].

Reimann, Anna (2015): “Ermittlungen gegen türkische Zeitung 'Cumhuriyet'”. Spiegel Online 15.01.15. http://www.spiegel.de/politik/ausland/charlie-hebdo-nachdruck-ermittlungengegen-tuerkische-zeitung-a-1013182.html [15.01.2015].

Siedlecka, Ewa (2014): „Kobieta z brodą - dla tolerancji”. Wyborcza 03.06.14. http://wyborcza.pl/1,134154,16082818,Kobieta_z_broda__dla_tolerancji.html [15.01.2015].

Thimm, Katja (2014): "Der dritte Weg". Der Spiegel 1/15. http://www.spiegel.de/spiegel/print/d-131045318.html [15.01.2015].

Titz, Christoph et al. (2014): "Kirchen lehnen "sexuelle Vielfalt" im Unterricht ab". Spiegel Online 10.01.14. http://www.spiegel.de/schulspiegel/homosexualitaet-im-unterrichtdebatte-weitet-sich-aus-a-942877.html [15.01.2015]. 
Töpper, Verena (2015): "Dresdner verhindern Einrichtung eines Flüchtlingsheims". Spiegel Online 14.01.15. http://www.spiegel.de/politik/deutschland/protest-gegen-asylbewerber-indresden-verhindert-fluechtlingsheim-a-1012846.html [15.01.2015].

Trautsch, Matthias (2015): "Keine Einigung auf Burka-Verbot". Frankfurter Allgemeine 21.01.15. http://www.faz.net/aktuell/rhein-main/frankfurt/burka-verbot-in-deutschlandnoch-nicht-entschieden-13381878.html [02.03.2015].

Ulrich, Stefan (2015): „Tapfere Spötter“. Süddeutsche Zeitung 15.01.15. http://www.sueddeutsche.de/kultur/anschlag-auf-charlie-hebdo-tapfere-spoetter-1.2303449 [17.01.2015].

Weber, Nina (2013): „Wurzel-Gen schützt Reis in Dürrezeiten“. Spiegel Online 05.08.13. http://www.spiegel.de/wissenschaft/natur/wurzel-gen-schuetzt-reis-in-duerrezeiten-a914540.html [15.01.2015]. 